\title{
Estimation of the Effective Permeability of Mixed-Matrix Membranes via Monte Carlo Simulations
}

\author{
Zheng Cao, Haoyu Wu, Boguslaw Kruczek and Jules Thibault \\ Department of Chemical and Biological Engineering \\ University of Ottawa, Ottawa, Canada K1N 6N5 \\ zcao088@uottawa.ca; hwu055@uottawa.ca; bkruczek@uottawa.ca; Jules.Thibault@uottawa.ca
}

\begin{abstract}
Mixed matrix membranes (MMMs), where dispersed inorganic fillers are embedded into the matrix of a polymer, have become the focus for research and development for a myriad of applications in gas separation and pervaporation. MMMs have spurred the interest of researchers in taking advantage of the inherent characteristics of polymer and inorganic fillers. The judicious choice of the fillers, pure or functionalized, can modify the membrane for a specific objective, such as enhancing the sorption of a targeted molecule, increasing or reducing the permeability of some species, or improving the mechanical strength of the membrane. This investigation is mainly concerned with the impact of the filler on the effective permeability of MMMs.

Gases permeating through a polymeric membrane usually is represented by the solution-diffusion model. In MMMs, the transport of gases across a membrane is represented by the effective solubility, effective diffusivity, and effective permeability. To estimate the effective permeability of MMMs, our group has traditionally used the finite difference (FD) method to solve the Fick's second law of diffusion. This FD method provides accurate results, but the computation time to achieve steady state tends to be enormous. To obtain more quickly the solution of Fick's second law of diffusion, we have resorted to Monte Carlo (MC) simulations. MC method has shown to be simple to implement and straightforward to characterize the properties of some complex membranes, including MMMs. For our particular application, the MC method uses stochastic methods to represent the Brownian motion of a species migrating through a MMM. This approach has proven accurate and efficient in estimating membrane properties, including solubility, permeability, diffusivity and even selectivity. For ideal MMMs with impermeable and permeable particles, MC simulations provide accurate results when compared to the published mathematical models and experimental data. Based on the simulation results, the effect of the fillers' aspect ratio, size, and shape on the effective permeability of the MMM has been analysed. All results are comparable to those obtained by the FD method but at an insignificant fraction of the computation cost.

The Monte Carlo approach provides a swift and reliable method to determine the transport properties of MMMs and can greatly assist in the design and performance evaluation of these membranes.
\end{abstract}

Keywords: Monte Carlo simulation; mixed-matrix membranes; gas separation; numerical modelling; membrane structure 